Physics

Physics Research Publications

\title{
Harmonic peaks of stripe phases in cuprates
}
B. Phillabaum
E. W. Carlson 


\title{
Quantum phase slips in a confined geometry
}

\author{
S. Khlebnikov \\ Department of Physics, Purdue University, West Lafayette, Indiana 47907, USA
}

(Received 13 September 2007; published 14 January 2008)

\begin{abstract}
We consider tunneling of vortices across a superconducting film that is both narrow and short (and connected to bulk superconducting leads at the ends). We find that in the superconducting state the resistance, at low values of the temperature $(T)$ and current, does not follow the power-law dependence on $T$ characteristic of longer samples but is exponential in $1 / T$. The coefficient of $1 / T$ in the exponent depends on the length or, equivalently, the total normal-state resistance of the sample. These conclusions persist in the one-dimensional limit, which is similar to the problem of quantum phase slips in an ultranarrow short wire.
\end{abstract}

DOI: 10.1103/PhysRevB.77.014505

PACS number(s): 74.78.Na, 74.40.+k

\section{INTRODUCTION}

Vortex tunneling across narrow thin films is a quantum effect that limits superconductivity in these systems. It does not rely on any vortices preexisting in the sample (due, e.g., to temperature or a magnetic field) but occurs as a result of quantum fluctuations-either a vortex entering the sample from the outside or a virtual vortex-antivortex pair created in the sample. A supercurrent (if present) exerts a force on the vortex and, for certain types of vortex motion, the total work done by this force is nonzero. That means that an amount of energy is taken away from the supercurrent, i.e., there is dissipation-an electrical resistance.

Frequently, one considers vortex tunneling at a strong enough current, so that the vortex can nucleate close to the boundary: the closer it nucleates, the shorter is the distance it has to tunnel, and the larger is the tunneling rate. ${ }^{1,2}$ However, for very narrow samples (starting perhaps with a few tens of nanometers), the rate may remain substantial even at weak currents, when the vortex tunnels the entire width. This is the case we consider here, motivated in part by its similarity to quantum phase slips in a genuinely one-dimensional (1D) wire, a topic of much current experimental research. ${ }^{3-13}$

While the core of a vortex can for our purposes be considered pointlike, it is important to take into account the long-range disturbance that vortex motion produces. Lowdimensional superconductors support a gapless plasmon mode, ${ }^{14,15}$ which in the presence of a nearby ground plate (the case considered here) has an acoustic dispersion law, with a speed $c_{0}$. This leads to a length scale, $l_{p}=\hbar c_{0} / T(T$ is the temperature), to which other length scales can be compared. In particular, one can distinguish between long wires, those of length $L \gg l_{p}$, for which the final state of plasmons does not depend on the boundary conditions at the ends, and short ones, $L \ll l_{p}$, for which it may.

For long 1D wires, plasmon production has been shown theoretically to significantly affect the tunneling rate. ${ }^{16-18}$ In the presence of disorder, it leads to a power-law currentvoltage dependence in the superconducting state at low temperatures, ${ }^{18} V(I) \propto I^{\alpha}$ (a state is superconducting if $\alpha>1$ ). Here, we consider the opposite, short-wire limit. (We will often use the system of units with $\hbar=c_{0}=1$, in which the short-wire condition is simply $L T \ll 1$.) This choice is motivated by a puzzle in the existing experimental results: while in a long wire the observed nonlinear $V(I)$ curve is indeed well described by a power law, ${ }^{11}$ in short wires, no power law in current or in temperature has been detected. ${ }^{10}$ Our results provide an explanation for that.

A general theory that allows one to compute the effect of plasmons on vortex tunneling for samples of various sizes can be constructed along the following lines. As well known (and reviewed, for example, in Ref. 19), two-dimensional superfluids have a dual description, in which vortices are viewed as charges and plasmons as "photons," so that the theory maps onto planar quantum electrodynamics (QED). We present a derivation of this, based on a path-integral identity, in Sec. II.

The version of QED that we use in this paper is "quenched," in the sense that it considers only a single vortex and neglects interaction with additional vortices that may tunnel nearby. We expect this to be a good approximation as long as one stays away from a superconductor-insulator transition. (For 1D wires, a mean-field-type theory that takes into account interactions between quantum phase slips has been recently proposed in Ref. 20.) In the quenched limit, the computation of the plasmon action amounts essentially to a Euclidean (imaginary time) version of the classical radiation theory. (The imaginary time appears since we are considering a tunneling process.) An important aspect of the theory is formulation of the boundary conditions at the ends of the sample. This is described in Sec. III, and the solution for the plasmon field is given in Sec. IV.

Our final result is that, in the superconducting state of narrow short wires connected to bulk superconducting leads, the resistance due to vortex tunneling, at small temperatures and currents, is no longer a power law but an exponential in $1 / T$. We trace this stronger suppression to a large gradient energy that the system must have already when it enters the classically forbidden region. The coefficient of $1 / T$ in the exponent depends inversely on the length $L$.

We wish to reiterate that this result applies only in the superconducting state, where the tunneling events (instantons) are rare, and does not preclude the possibility of a transition to an insulating state at larger tunneling rates.

The tunneling process we consider here is in addition to and competes with the classical, overbarrier process. The latter is an analog of a thermally activated phase slip in the 1D case. ${ }^{21-23}$ As we will see, despite the above-mentioned sup- 
pression, the rate of vortex tunneling is exponentially larger than the rate of thermal activation over a broad range of parameters. We hope that the difference in both the magnitudes of the resistance and its dependence on the length will allow one to distinguish between the two effects experimentally.

We should also note the difference in the starting points for the theories of these two effects. The energy of the LAMH saddle point ${ }^{22,23}$ is due to the phase slip core (and consequently depends strongly on the Ginzburg-Landau coherence length $\xi$ ). In contrast, in our case, the activation energy is that of the initial tunneling state, which lies far from the top of the potential barrier. Depletion of the order parameter in this state is still small, and we can use the phase-only theory, in which vortex cores are essentially pointlike.

\section{DUALITY MAP}

The Lagrangian density of the phase-only theory that describes a superconducting film in the presence of a nearby ground plate is

$$
\mathcal{L}=\frac{1}{2 g}\left(\partial_{t} \theta\right)^{2}-\frac{1}{2} K_{s}(\nabla \theta)^{2}=\frac{1}{2} K_{s} \partial^{\mu} \theta \partial_{\mu} \theta .
$$

In the second equality here we have switched to the notation of special relativity, by using instead of time $t$ the coordinate $x^{0}=c_{0} t$, where $c_{0}=\sqrt{g K_{s}}$ is the plasmon speed. (In addition, $x^{1} \equiv x$ and $x^{2} \equiv y$.) In what follows, we choose units of length and time so that $c_{0}=1$. Greek indices take values 0,1 , and 2 , and summation over a repeated index is implied.

The field $\theta$ is the phase of the order parameter, but no assumption is made about the existence of long-range order, i.e., we do no require the expectation value of $\exp (i \theta)$ to be nonzero. All that is required for superfluidity is that the stiffness $K_{s}$ renormalizes to a nonzero value in the infrared.

If $\theta$ were a single-valued smooth function of $(x, y)$, plasmons would be the only excitations in the system, and the theory would be completely Gaussian. To describe vortices, we allow $\theta$ to be multivalued. Alternatively, we could make it discontinuous by drawing explicit branch cuts at vortex positions, but we will be using the first approach. Then, $\nabla \theta$ is smooth outside vortex cores.

The phase-only description (1) does not resolve vortex cores, so a short-scale cutoff of order of the GinzburgLandau coherence length $\xi$ is implied.

Let us remark on the issue of gauge invariance and the apparent absence of electromagnetic fields from Eq. (1). In thin films, magnetic field of a vortex extends over a large area, determined by the transverse screening length $\lambda_{\perp} \cdot{ }^{24}$ If $\lambda_{\perp}$ (which in thin films can exceed $100 \mu \mathrm{m}$ ) is much larger than the smallest dimension of the film, the magnetic field can be neglected, and it is possible to choose a gauge such that the vector potential $\mathbf{A}$ is close to zero. The remaining gauge freedom can be used to make the scalar potential $A_{0}$ go to zero away from the film. This fixes the gauge completely, and if $\theta$ denotes the phase in this gauge it is in effect gauge invariant. Integrating out $A_{0}$ produces a capacitive term, which eventually becomes the first term in Eq. (1).

We have not included in Eq. (1) a "topological" term, proportional to $\partial_{t} \theta$, that gives rise to the Magnus force on a vortex. We consider films that have significant amounts of disorder, and in disordered superconductors the Magnus force is small. ${ }^{25}$

Equation (1) allows one to describe dissipation of energy of vortex motion into plasmons, but does not include any dissipative mechanisms related to normal electrons at the vortex cores. This is justified in the limit of strong disorder, since transfer of energy to the normal component in this case is inhibited by the short electron mean-free path.

Because in the presence of vortices $\theta$ is multivalued, the expression

$$
J^{\mu}=\frac{1}{2 \pi} \epsilon^{\mu \nu \lambda} \partial_{\nu} \partial_{\lambda} \theta,
$$

where $\epsilon^{\mu \nu \lambda}$ is the unit antisymmetric tensor, is nonzero, and indeed is the vortex current $\left(J^{0}\right.$ is the vortex density). Setting

$$
q_{\lambda} \equiv \partial_{\lambda} \theta
$$

we can write the current (2) as

$$
J^{\mu}=\frac{1}{2 \pi} \epsilon^{\mu \nu \lambda} \partial_{\nu} q_{\lambda}
$$

The equation of motion following from Eq. (1) is

$$
\partial^{\lambda} \partial_{\lambda} \theta=\partial^{\lambda} q_{\lambda}=0
$$

[In the presence of a topological term, the temporal component of Eq. (3) is replaced by $q_{0}=\partial_{0} \theta+$ const, and Eq. (11) below is modified accordingly. The expression (4) for the current and the equation of motion (5) are both unaffected.]

In what follows we restrict our attention to configurations satisfying the equation of motion (5). In the real-time version of the theory, they describe motion of an arbitrary number of real vortices in the presence of supercurrents and plasmon waves. In the Euclidean (imaginary time) version, to which we turn shortly, solutions to the equations of motion will determine the most probable tunneling paths (instantons) responsible for the quantum decay of supercurrents.

The real-time action corresponding to the Lagrangian density (1) is

$$
S=\frac{1}{2} K_{s} \int \partial^{\mu} \theta \partial_{\mu} \theta d^{3} x=\frac{1}{2} K_{s} \int q^{\mu} q_{\mu} d^{3} x,
$$

where $d^{3} x=d x^{0} d x^{1} d x^{2}$. If $q_{\mu}$ is a solution of the equation of motion (5), there is a path-integral identity - the duality map

$$
\begin{aligned}
e^{i S}= & \int \mathcal{D} f_{\mu \nu} \mathcal{D} \lambda \exp \int i K_{S}\left\{-\frac{1}{4} f^{\mu \nu} f_{\mu \nu}-\frac{1}{2} \epsilon^{\mu \nu \rho} f_{\mu \nu} q_{\rho}\right. \\
& \left.+\frac{1}{2} \lambda \epsilon^{\mu \nu \rho} \partial_{\rho} f_{\mu \nu}\right\} d^{3} x,
\end{aligned}
$$

where $f_{\mu \nu}$ is antisymmetric in $\mu, \nu$ and is subject to the boundary condition 


$$
\left.\epsilon^{\mu \nu \rho} f_{\mu \nu} n_{\rho}\right|_{b}=-2 n^{\rho} q_{\rho} ;
$$

$n_{\rho}$ is the normal to the boundary $(b)$ of the space-time volume.

Because the path integral in Eq. (7) is Gaussian, the map can be verified directly. First, note that the path integral over $\lambda$ enforces the Bianchi identity

$$
\epsilon^{\mu \nu \rho} \partial_{\rho} f_{\mu \nu}=0 .
$$

Since in Eq. (7) $f_{\mu \nu}$ is an independent variable and not (yet) a curl of some gauge field, Eq. (9) is not really an identity but an independent equation of motion; however, we keep the familiar term.

Next, integrating over $f_{\mu \nu}$ amounts to solving the saddlepoint equation

$$
f^{\mu \nu}+\epsilon^{\mu \nu \rho}\left(q_{\rho}+\partial_{\rho} \lambda\right)=0 .
$$

Taking curl of this and using Eqs. (9) and (5), we obtain $\partial^{\rho} \partial_{\rho} \lambda=0$. On the other hand, applying the boundary condition (8) in Eq. (10) tells us that the normal derivative of $\lambda$ at the boundary is zero. For a space-time volume of a simple shape, these conditions are sufficient to reduce $\lambda$ to a constant, which then drops out of Eq. (10). Equation (10) becomes

$$
f^{\mu \nu}=-\epsilon^{\mu \nu \rho} q_{\rho}=-\epsilon^{\mu \nu \rho} \partial_{\rho} \theta .
$$

Substituting this back in Eq. (7) we confirm that the original action (6) is recovered. In terms of the saddle-point value (11), this action can be written as

$$
S=\frac{1}{4} K_{s} \int f^{\mu \nu} f_{\mu \nu} d^{3} x .
$$

Now, let us take an alternative ("dual") view and solve the identity (9) explicitly by introducing a gauge field $a_{\mu}$ (quite distinct from the electromagnetic potential $A_{\mu}$ ) as follows:

$$
f_{\mu \nu}=\partial_{\mu} a_{\nu}-\partial_{\nu} a_{\mu}
$$

Differentiating Eq. (11), we obtain the following Maxwell equations for this field:

$$
\partial_{\nu} f^{\mu \nu}=-2 \pi J^{\mu}
$$

where $J^{\mu}$ is the vortex current (4). The usefulness of this dual view is that it allows one to determine $f_{\mu \nu}$, and the corresponding action, for any prescribed motion of vortices. Using Eq. (11), one can then find the derivatives of the phase $\theta$.

The dual theory also allows one to take into account the backreaction of produced photons on the vortex motion, by extremizing the total action with respect to that motion itself. In the problem to which we apply this theory here, backreaction will determine the region where the vortex prefers to tunnel. (This region turns out to be the middle of the wire-in contrast to the resistively shunted 1D case, where quantum phase slips occur preferentially near the ends. ${ }^{26}$ )

To any solution of Eq. (14) we can add a homogeneous solution-a static uniform "electric" field $f_{0 i}=$ const. According to Eq. (11), such a field corresponds to a static uniform supercurrent. We adopt the convention in which the supercurrent density is measured in units of $-2|e|(e$ is the elec- tron charge), i.e., is given by $K_{s} \nabla \theta$. Then, for example, a current in the positive $x$ direction corresponds to an "electric" field in the negative $y$ direction, and if a vortex moves that way, far from any boundaries, the work done on it by the current will be positive. In the presence of boundaries, a nontrivial change of $\theta$ at a boundary may give an additional contribution to the total work.

\section{RADIATION THEORY}

To describe tunneling, we switch to the Euclidean time, via

$$
\begin{gathered}
\tau=i t, \\
a_{4}=-i a_{0}, \\
J^{4}=i J^{0} .
\end{gathered}
$$

The relations (11) take the form

$$
\begin{gathered}
-i \partial_{4} \theta=f_{x y} \equiv B, \\
i \partial_{y} \theta=f_{x 4} \equiv E, \\
-i \partial_{x} \theta=f_{y 4} \equiv F,
\end{gathered}
$$

where we have introduced shorthands $B, E$, and $F$, which will be much used in what follows.

The Euclidean counterpart of the action (12) is

$$
S_{E}=-\frac{1}{2} K_{s} \int d x d y d \tau\left(B^{2}+E^{2}+F^{2}\right) .
$$

Note that, as a result of the transition to the Euclidean time, the relations of all three components of $f_{\mu \nu}$ to the derivatives of $\theta$ have acquired factors of $i$. As a consequence, on the instanton solution, $B, E$, and $F$ will all be purely imaginary, and the action (18) will be positive. This is in agreement with the a priori expectation that coupling to plasmons should suppress vortex tunneling (the suppression factor is $e^{-S_{E}}$ ) since, as it tunnels, the vortex has to drag the plasmon subsystem with it.

Maxwell equations (14) in these notations have the form

$$
\begin{gathered}
-\partial_{4} E-\partial_{y} B=2 \pi J^{x}, \\
-\partial_{4} F+\partial_{x} B=2 \pi J^{y}, \\
\partial_{x} E+\partial_{y} F=2 \pi J^{4},
\end{gathered}
$$

while the Bianchi identity reads

$$
\partial_{x} F=-\partial_{4} B+\partial_{y} E .
$$

Using these together, one can obtain independent wave equations for $B, E$, and $F$. All that is left to choose, then, is a suitable form of the vortex current and the boundary conditions for the fields.

We consider the theory on a rectangular strip of length $L$ $(0 \leq x \leq L)$ and width $w(0 \leq y \leq w)$ and assume that the vor- 


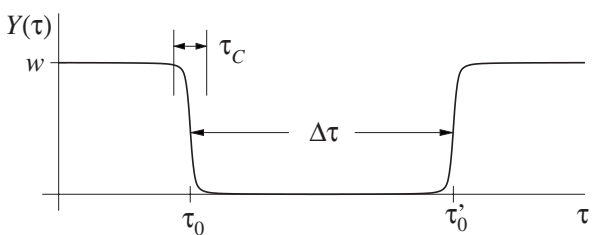

FIG. 1. The vortex's transverse position as a function of the Euclidean time. $\Delta \tau$ denotes the instanton-anti-instanton separation, and $\tau_{C}$ the duration of an instanton. In the text, we also use the rescaled variable $\tilde{Y}=Y / w$, where $w$ is the wire's width.

tex motion is purely transverse: $J^{x}=0$. Then, the wave equations are

$$
\begin{aligned}
& \partial_{4}^{2} B+\nabla^{2} B=2 \pi \partial_{x} J^{y}, \\
& \partial_{4}^{2} E+\nabla^{2} E=2 \pi \partial_{x} J^{4} .
\end{aligned}
$$

Once solutions to these are obtained, the solution for $F$ can be found from Eq. (20) or Eq. (21), except for the static uniform component. The latter is the static uniform "electric" field mentioned at the end of Sec. II. It corresponds to a steady supercurrent in the $x$ direction, and that supercurrent can in principle have any value. Eventually, this component of $F$ will be determined by the properties of the metastable state from which the system tunnels.

The remaining (nonzero) components of the vortex current are

$$
\begin{gathered}
J^{y}=i \partial_{\tau} Y \delta(x-X) \delta(y-Y(\tau)), \\
J^{4}=i \delta(x-X) \delta(y-Y(\tau)),
\end{gathered}
$$

where $Y(\tau)$ is the transverse position of the vortex. At a finite temperature $T, Y(\tau)$ must be periodic in $\tau$ with period $\beta$ $=1 / T$.

If the vortex could nucleate inside the strip, $Y(\tau)$ would start at the upper edge, $Y=w$, move down to $Y=Y_{\text {nucl }}$, the nucleation point, and then back to $Y=w$. This would form a "bounce." 27 As we already noted, though, here we consider only supercurrents that are small enough for the vortex to have to tunnel the entire width $w$. Then, the relevant configuration is an instanton-anti-instanton (IA) pair: a vortex tunneling across the strip around time $\tau=\tau_{0}$, plus an antivortex (or a vortex moving in the opposite direction) tunneling around $\tau=\tau_{0}^{\prime}$. A representative history of $Y(\tau)$ is, shown in Fig. 1.

Turning to the boundary conditions (b.c.'s), we note that, since there is no current through the edges of the strip, $B$ and $E$ satisfy, respectively, the Neumann and Dirichlet boundary conditions at $y=0$ and $w$. We can then define Fourier transforms with respect to $y$ and $\tau$ as follows:

$$
B(x, y ; \tau)=T \sum_{l n} e^{-i \Omega_{n} \tau} \psi_{l}(y) B_{l n}(x),
$$

$$
E(x, y ; \tau)=T \sum_{l n} e^{-i \Omega_{n} \tau} \chi_{l}(y) E_{l n}(x),
$$

where $\psi_{l}(y)=\cos (\pi l y / w)$ and $\chi_{l}(y)=\sin (\pi l y / w)$, and the sum over $l$ starts from $l=0$ in the first case and from $l=1$ in the second; $\Omega_{n}=2 \pi n T$ are the Matsubara frequencies. In either case, the wave operator takes the form

$$
\partial_{4}^{2}+\nabla^{2} \rightarrow \partial_{x}^{2}-k_{l n}^{2}
$$

with

$$
k_{l n}^{2}=\frac{\pi^{2} l^{2}}{w^{2}}+\Omega_{n}^{2}
$$

The action (18) becomes

$$
S_{E}=-\frac{1}{2} K_{s} w T \sum_{l n} C_{l} \int_{0}^{L} d x\left(B_{l n} B_{l,-n}+E_{l n} E_{l,-n}+F_{l n} F_{l,-n}\right),
$$

where $C_{l}=1$ for $l=0$ and $C_{l}=\frac{1}{2}$ otherwise.

To obtain the b.c. at $x=0, L$, we need to specify how the sample connects to the outside world. Here, we consider the case when the leads are bulk superconductors. As a model of those, we use strips of the same width $w$ and with same parameter $g$ as the wire but of much larger stiffness, $K_{s}^{\prime}$ $\gg K_{s}$, and length $L^{\prime} \gg L$. $L^{\prime}$ will eventually be taken to infinity.

Consider the interface at $x=L$. Denote the Fourier components of $\theta$ there as

$$
\theta_{l n}(L) \equiv \bar{\theta}_{l n}
$$

Then, for any $l$ and $n$ that are not both zero, throughout the lead $(x>L)$

$$
\theta_{l n}(x)=\bar{\theta}_{l n} e^{-k_{l n}^{\prime}(x-L)},
$$

where $k_{l n}^{\prime}>0$ and is given by Eq. (26) with $\Omega_{n}^{2}$ replaced by $\Omega_{n}^{2} / g K_{s}^{\prime}$. Substituting Eq. (28) into the action of the lead, we obtain a contribution to the effective action of $\bar{\theta}_{l n}$ as follows:

$$
S_{E}^{\prime}=\frac{1}{2} K_{s}^{\prime} w T \sum_{k_{l n}^{\prime}>0} C_{l} k_{l n}^{\prime} \bar{\theta}_{l n} \bar{\theta}_{l,-n}
$$

When we extremize the total action with respect to $\bar{\theta}_{l n}$, this term gives $\bar{\theta}_{l,-n}$ with a coefficient that grows at least as $\sqrt{K_{s}^{\prime}}$ at large $K_{s}^{\prime}$. As a result, at large $K_{s}^{\prime}, \bar{\theta}_{l n}$ are close to zero. The same applies at the other interface, at $x=0$.

We conclude that, in the case of bulk superconducting leads, both $B$ and $E$ satisfy the Dirichlet b.c. at either end,

$$
\begin{aligned}
& B_{l n}(0)=B_{l n}(L)=0, \\
& E_{l n}(0)=E_{l n}(L)=0
\end{aligned}
$$

$\left(k_{l n}>0\right)$. The absence of $k_{l n}=0$ from these conditions is inconsequential, since neither $B$ nor $E$ has an $l=n=0$ mode (only $F$ does).

Note that the condition (29) is not satisfied by the trial instanton configurations considered (for the 1D case) in Ref. 
28. This explains the difference in the final results: Ref. 28 finds that the phase slip rate remains finite at $T \rightarrow 0$, while we find that it is exponential in $1 / T$.

We now proceed to solving the wave equations for $B$ and $E$, and determining $F$ and the Euclidean action.

\section{SOLUTION FOR THE PLASMON}

Since the current components (24) and (25) have the same $x$ dependence, both $B$ and $E$ can be expressed through solutions to the equation

$$
-\partial_{x}^{2} f_{l n}+k_{l n}^{2} f_{l n}=\partial_{x} \delta(x-X)
$$

$\left(k_{l n}>0\right)$ with the Dirichlet b.c. $f_{l n}(0)=f_{l n}(L)=0$. These solutions are readily found,

$$
f_{l n}(x)=\frac{1}{\sinh \left(k_{l n} L\right)}\left\{\begin{array}{cc}
\cosh \left[k_{l n}(L-X)\right] \sinh \left(k_{l n} x\right), & x<X \\
-\cosh \left(k_{l n} X\right) \sinh \left[k_{l n}(L-x)\right], & x>X,
\end{array}\right.
$$

although, as we will see, many useful conclusions can be drawn even without using this explicit form.

From now on, we assume that the width $w$ is much smaller than the length $L$ (i.e., $w \ll L$ ) and restrict attention to the $l=0$ ( $y$-independent) modes, which are the only ones that are potentially infrared sensitive. For tunneling paths such as the one shown in Fig. 1, this makes our problem similar to the problem of a quantum phase slip in a genuinely $1 \mathrm{D}$ wire.

The field $E$ has no $l=0$ modes, so it drops out of the subsequent discussion. The other fields are

$$
\begin{gathered}
B_{0 n}(x)=-2 \pi i\left(\partial_{\tau} \tilde{Y}\right)_{n} f_{0 n}(x), \\
F_{0 n}(x)=\frac{2 \pi}{\Omega_{n}}\left(\partial_{\tau} \tilde{Y}\right)_{n}\left[\partial_{x} f_{0 n}(x)+\delta(x-X)\right], \quad n \neq 0,
\end{gathered}
$$

where $\tilde{Y}$ is the rescaled transverse coordinate, $\tilde{Y}=Y / w$, and $\left(\partial_{\tau} \tilde{Y}\right)_{n}$ is the Fourier transform of $\partial_{\tau} \tilde{Y}$,

$$
\left(\partial_{\tau} \tilde{Y}\right)_{n}=\int_{0}^{\beta} d \tau \partial_{\tau} \tilde{Y} e^{i \Omega_{n} \tau}=-i \Omega_{n} \widetilde{Y}_{n} .
$$

The $n=0$ component of $B$ is zero, while that of $F$ is the static uniform component that should be determined from the properties (the winding number) of the metastable state.

Let us take up the latter task first. In the wire, set $F_{00}$ $=-i I / K_{s} w T$, where $I$ needs to be determined. Then, in the leads, $F_{00}=-i I / K_{s}^{\prime} w T$. The total winding number is

$$
N(\tau)=i \int_{-L^{\prime}}^{L+L^{\prime}} F(x, \tau) d x=\frac{I}{w}\left(\frac{L}{K_{s}}+\frac{2 L^{\prime}}{K_{s}^{\prime}}\right)+2 \pi\left[\tilde{Y}(\tau)-T \tilde{Y}_{0}\right] .
$$

The last term here is due to the $n \neq 0$ modes (34),

$$
\begin{aligned}
i T \sum_{n \neq 0} e^{-i \Omega_{n} \tau} \int_{0}^{L} F_{0 n}(x) d x & =2 \pi T \sum_{n \neq 0} e^{-i \Omega_{n} \tau} \tilde{Y}_{n} \\
& =2 \pi\left[\tilde{Y}(\tau)-T \tilde{Y}_{0}\right] .
\end{aligned}
$$

Note that the sum evaluates not to $\tilde{Y}(\tau)$ but to $\tilde{Y}(\tau)$ without the zero mode. As a result, $N$ at a given time depends on the entire history of $\tilde{Y}(\tau)$, in particular, on the value of the IA separation $\Delta \tau$.

The initial and final states of tunneling correspond to points midway between the instanton and anti-instanton. These are the points at which the system enters and leaves the classically forbidden region. If the instanton and antiinstanton positions are sharply defined, i.e., $\tau_{C} \ll \Delta \tau$ (cf. Fig. $1)$, the corresponding times are

$$
\begin{gathered}
\tau_{f}=\frac{1}{2}\left(\tau_{0}+\tau_{0}^{\prime}\right), \\
\tau_{i}=\tau_{f}-1 / 2 T .
\end{gathered}
$$

The winding numbers at these times can be computed from Eq. (36) and compared to those of ground states with uniform supercurrents.

In particular, the winding number at $\tau=\tau_{i}$ is the same as in the uniform ground state with supercurrent $I_{g s}$ given by

$$
\frac{I_{g s}}{w}\left(\frac{L}{K_{s}}+\frac{2 L^{\prime}}{K_{s}^{\prime}}\right)=N\left(\tau_{i}\right)
$$

Thus, the initial state belongs to the thermal ensemble built near that ground state. A similar relation (with a different ground-state current) applies in the final state, and we find, as expected, that the instanton describes tunneling between two thermal ensembles that differ by a $2 \pi$ of the winding number.

The duration of an individual instanton, $\tau_{C}$ in Fig. 1, is determined by the parameters of the sample. Meanwhile, as we will see, the IA separation $\Delta \tau$ is controlled by the values of the temperature and current and becomes large when those are small. So, for calculating Eq. (36), we can approximate $\partial_{\tau} \tilde{Y}$ in Eq. (35) as

$$
\partial_{\tau} \tilde{Y} \approx-\delta\left(\tau-\tau_{0}\right)+\delta\left(\tau-\tau_{0}^{\prime}\right) .
$$

Then, the last term in Eq. (36) becomes

$$
\tilde{Y}\left(\tau_{i}\right)-T \tilde{Y}_{0}=T \Delta \tau,
$$

where $\Delta \tau=\tau_{0}^{\prime}-\tau_{0}$. In the limit $L^{\prime} \rightarrow \infty$, Eq. (37) gives

$$
I_{g s}=I+\frac{\pi K_{s}^{\prime} w T \Delta \tau}{L^{\prime}} .
$$

This relation between $I$ and $I_{g S}$ can be used to learn how much action is contained in the $l=n=0$ mode. The main contribution comes from the leads, where $F_{00}=-i I / K_{s}^{\prime} w T$ and $F_{g s, 00}=-i I_{g s} / K_{s}^{\prime} w T$. So, the $l=n=0$ term in the action (27), relative to the corresponding term in the ground state, equals

$$
S_{0}=\frac{1}{2} K_{s}^{\prime} w T \int d x\left(\left|F_{00}\right|^{2}-\left|F_{g s, 00}\right|^{2}\right)=-2 \pi I \Delta \tau+O\left(1 / L^{\prime}\right) .
$$

Note that this is the only term in the action that distinguishes between direct and reverse processes: it would change sign if we considered an antivortex tunneling at $\tau=\tau_{0}$ (or a vortex tunneling in the opposite, positive $y$, direction). 
Turning to the $n \neq 0$ modes, given by Eq. (33) and (34), we find that their action is

$$
S_{1}=2 \pi^{2} K_{s} w T \sum_{n \neq 0} \int_{0}^{L} d x\left\{\Omega_{n}^{2} f_{0 n}^{2}+\left[\partial_{x} f_{0 n}+\delta(x-X)\right]^{2}\right\}\left|\tilde{Y}_{n}\right|^{2} .
$$

Integrating by parts in the derivative term and using Eq. (31), we bring this to the form

$$
S_{1}=2 \pi^{2} K_{s} w T \sum_{n \neq 0}\left\{\partial_{x} f_{0 n}(0)+\Omega_{n}^{2} \int_{0}^{X} f_{0 n}(x) d x\right\}\left|\tilde{Y}_{n}\right|^{2} .
$$

Using the explicit form of $f_{0 n}$, we find for the term in the braces

$$
\begin{aligned}
\partial_{x} f_{0 n}(0)+\Omega_{n}^{2} \int_{0}^{X} f_{0 n}(x)= & \frac{k_{n}}{\sinh \left(k_{n} L\right)} \cosh \left[k_{n}(L-X)\right] \\
& \times \cosh \left(k_{n} X\right),
\end{aligned}
$$

where $k_{n} \equiv\left|\Omega_{n}\right|$. This has a minimum at $X=L / 2$ (albeit a shallow one at small $k_{n}$ ), meaning that the vortex prefers to tunnel in the middle of the wire. Setting $X=L / 2$, we finally obtain

$$
S_{1}=\pi^{2} K_{s} w T \sum_{n \neq 0} k_{n} \operatorname{coth}\left(k_{n} L / 2\right)\left|\tilde{Y}_{n}\right|^{2}
$$

The approximation (38) correctly reproduces Fourier components of $\partial_{\tau} \tilde{Y}$ with $k_{n} \ll 1 / \tau_{C}$. For our purposes, it will be sufficient to consider only these $k_{n}$, so we can make the replacement

$$
\left|\tilde{Y}_{n}\right|^{2}=\frac{2}{\Omega_{n}^{2}}\left[1-\cos \left(\Omega_{n} \Delta \tau\right)\right]
$$

in Eq. (40) and cut off the sum in the ultraviolet at $k_{n}$ $=1 / \tau_{C}$.

The resulting expression for the Euclidean action, $S_{E}$ $=S_{0}+S_{1}$, is applicable both to short $(L T \ll 1)$ and to long $(L T \gg 1)$ wires. In the latter case, it reproduces the result of Ref. 18, obtained by considering phase slips directly in the one-dimensional theory. In what follows, we restrict attention to the former case.

We now need to determine $\Delta \tau$. We begin by considering the action (40) for different values of it. For

$$
|\Delta \tau| \ll 1 / T
$$

the sum in Eq. (40) can be approximated by an integral as follows:

$$
S_{1} \approx 2 \pi K_{s} w \int_{0}^{1 / \tau_{C}} \frac{d k}{k}[1-\cos (k \Delta \tau)] \operatorname{coth}(k L / 2) .
$$

We see that substantial contributions can only come from $k$ $\gtrsim 1 /|\Delta \tau|$. It is therefore convenient to separate the range (42) into two. For $|\Delta \tau| \ll L$, the cotangent can be replaced with unity, and we are back to the expression ${ }^{18}$ for the long-wire case. The underlying physics is that the typical wave number of plasmons in the final state is of order $1 /|\Delta \tau|$, and when this is much larger than $1 / L$ the effect of the boundaries is insignificant. In the present case, however, this is possible only for relatively large currents. Indeed, extremizing the total action $S_{0}+S_{1}$ with respect to $\Delta \tau$, we obtain the saddlepoint value $\Delta \tau=K_{s} w / I$. For this to be much smaller that $L$, we need $I \gg K_{s} w / L$. Although this condition does not look altogether prohibitive, from now on we concentrate on the opposite, small-current, regime

$$
I \ll \frac{K_{s} w}{L} .
$$

Then, there is no saddle point either for $|\Delta \tau| \ll L$ or for $|\Delta \tau| \sim L$.

As $|\Delta \tau|$ increases past $L$-while still obeying Eq. (42) the dependence of Eq. (43) on $|\Delta \tau|$ becomes linear, and one can verify that, for small currents, this again precludes a saddle point. So, we turn to $\Delta \tau \sim 1 / T$ and the general expression (40) for $S_{1}$.

Equation (40) has an extremum at $\Delta \tau=1 / 2 T$ (derivative of each term in the sum vanishes individually). This extremum is, in fact, a maximum, as it should be: the original integration was over real $\Delta t=-i \Delta \tau$, and a saddle point that is a minimum in the real $\Delta t$ direction is a maximum in real $\Delta \tau$. The contribution from $S_{0}$ will displace the maximum from exactly $1 / 2 T$, but for small currents this displacement is small, and for our purposes $\Delta \tau=1 / 2 T$ is a good approximation. Substituting it into Eq. (41), we find that only odd $n$ contribute, and $S_{1}$ becomes, to logarithmic accuracy,

$$
\begin{aligned}
S_{1} & =8 \pi^{2} K_{s} w T \sum_{n=1,3, \ldots}^{n_{C}} \frac{1}{k_{n}} \operatorname{coth}\left(k_{n} L / 2\right) \\
& \approx \frac{\pi^{2} K_{s} w}{2 L T}+2 \pi K_{s} w \ln \frac{L}{\tau_{C}},
\end{aligned}
$$

where $n_{C} \sim 1 / \tau_{C} T$. In what follows, we retain only the first, leading, term on the right-hand side.

The power dissipated by vortex tunneling is given by the energy $2 \pi I$ that a vortex releases from the supercurrent, times the difference between the rates of the direct and reverse processes as follows:

$$
P(I)=2 \pi I\left[\mathcal{R}_{+}(I)-\mathcal{R}_{-}(I)\right] .
$$

To the exponential accuracy, $\mathcal{R}_{+}(I) \sim \exp \left[-S_{0}(I)-S_{1}\right]$, and $\mathcal{R}_{-}(I)=\mathcal{R}_{+}(-I)$. Expanding in small $I$, we obtain the resistance

$$
R(T) \sim e^{-S_{1}} \sim \exp \left(-\frac{\pi^{2} K_{s} w}{2 L T}\right) .
$$

The activated behavior of the resistance can be interpreted by looking at the gradient of the phase $\theta$ at the entry point of tunneling, $\tau=\tau_{i}$. Using the approximation (41) and setting $\Delta \tau=1 / 2 T$, we obtain

$$
\begin{aligned}
\partial_{x} \theta\left(x, \tau_{i}\right)=i F\left(x, \tau_{i}\right)= & \frac{I}{K_{s} w}+4 \sum_{n=1,3, \ldots} \frac{1}{n} \sin (\pi n / 2)\left[\partial_{x} f_{0 n}(x)\right. \\
& +\delta(x-X)] .
\end{aligned}
$$

For low-frequency modes, those with $0<k_{n} \ll 1 / L$, 


$$
\partial_{x} f_{0 n}(x)+\delta(x-X) \approx 1 / L,
$$

so the total contribution of these modes to Eq. (46) is $\left(\partial_{x} \theta\right)_{\text {low }} \approx \pi / L$. Then, the gradient energy contained in these modes is

$$
\mathcal{E}=\frac{1}{2} K_{s} w \int_{0}^{L}\left(\partial_{x} \theta\right)_{\mathrm{low}}^{2} d x \approx \frac{\pi^{2} K_{s} w}{2 L}
$$

in precise accord with Eq. (45). This means that the main effect suppressing resistance at low temperature is the population of the initial tunneling state, due to the large gradient of $\theta$ already required by that time. There is, of course, an additional action associated with the tunneling itself, but the precise agreement between Eqs. (45) and (47) implies that it is only subleading.

We should note that the entirely classical appearance of the exponent in Eq. (45) (it does not require any powers of $\hbar$ ) does not contradict it being a consequence of tunneling, rather than a classical, overbarrier process. Indeed, restoring $\hbar$ and $c_{0}$ in the short-wire condition, under which Eq. (45) applies, we obtain $T \ll \hbar c_{0} / L$, and this cannot be realized outside of quantum mechanics.

For comparison, let us list nucleation energies for two purely classical processes. The nucleation energy of a vortex is of order $\pi K_{s}$ (times a logarithm), and that of the $y$-independent saddle point, analogous to the LAMH saddle point in 1D wires, ${ }^{22,23}$ is of order $K_{s} w / \xi$, where $\xi$ is the Ginzburg-Landau coherence length. As long as $w$ and $\xi$ are both much smaller than $L$, either of these energies is larger than the energy (47).

Due to a relatively large numerical factor $\left(\pi^{2} / 2\right)$ in Eq. (47), the condition $\xi \ll L$, under which the energy (47) is smaller than the LAMH activation energy, may, in fact, mean that $L$ must be several times larger than $\xi$. Moreover, even if this condition is satisfied at low temperatures, it breaks down in a region close to the critical temperature $T_{c}$. The shortwire condition also breaks down near in a region near $T_{c}$, since $K_{s}$ and hence $c_{0}$ are small there; in that region, subleading terms in the instanton action become non-negligible. Outside of these regions, however, vortex tunneling is the dominant resistive process.

For a superconductor in the dirty limit, the exponent in Eq. (45) can be expressed entirely in terms of the superconducting gap $\Delta \equiv \Delta(T)$ and the normal-state resistance $R_{N}$ $=\rho L / w$, where $\rho$ is the sheet resistivity. Indeed, in this case $K_{s}=\left(\pi \Delta / 4 e^{2} \rho\right) \tanh (\Delta / 2 T),{ }^{29}$ so

$$
R(T) \sim \exp \left(-\frac{\pi^{2} R_{q} \Delta}{4 R_{N} T} \tanh \frac{\Delta}{2 T}\right),
$$

where $R_{q}=\pi / 2 e^{2}=6.5 \mathrm{k} \Omega$. This suggests that the value $R_{N}$ $=R_{q}$ may have a special significance in short wires. Experimentally, it does: this is the value near which one observes a superconducting-insulating transition. ${ }^{6,13}$

\section{CONCLUSION}

We have described a general method for calculating the effect of plasmons on vortex tunneling in superconducting wires and applied it to the limit of small temperatures and currents, when the vortex has to tunnel the entire width of the wire. The method is based on a duality map, through which vortices become charges and plasmons become "photons." We have found that, if plasmons cannot easily leave the tunneling region, as is the case when the wire is short and the leads are bulk superconductors, the suppression of the resistance in the superconducting state is exponential at low temperatures and expressed by Eq. (45).

As the width of the sample is made smaller and approaches the coherence length $\xi$, vortex tunneling crosses over to quantum phase slips in a genuinely $1 \mathrm{D}$ geometry. The restriction to modes independent of $y$ (the transverse coordinate) that we made in Sec. IV effectively brings us one dimension down, so we expect the $1 \mathrm{D}$ case to be similar to ours.

Despite the exponential suppression, the resistance due to (thermally assisted) vortex tunneling is larger, over a broad range of parameters, than that due to classical, overbarrier processes, such as motion of a thermally nucleated vortexantivortex pair or a thermally activated phase slip. In addition, it has a characteristic dependence on the length $L$ of the sample or, equivalently, on the total normal-state resistance $R_{N}$, cf. Eq. (48). We hope that these features will allow one to distinguish between the quantum and classical processes in the experiment.

\section{ACKNOWLEDGMENT}

The author thanks A. Bezryadin for useful comments.
${ }^{1}$ L. I. Glazman and N. Ya. Fogel, Fiz. Nizk. Temp. 10, 94 (1984) [Sov. J. Low Temp. Phys. 10, 51 (1984)].

${ }^{2}$ F. Tafuri, J. R. Kirtley, D. Born, D. Stornaiuolo, P. G. Medaglia, P. Orgiani, G. Balestrino, and V. G. Kogan, Europhys. Lett. 73, 948 (2006).

${ }^{3}$ N. Giordano, Phys. Rev. Lett. 61, 2137 (1988); Phys. Rev. B 41, 6350 (1990).

${ }^{4}$ N. Giordano and E. R. Schuler, Phys. Rev. Lett. 63, 2417 (1989).

${ }^{5}$ N. Giordano, Phys. Rev. B 43, 160 (1991); Physica B 203, 460 (1994).
${ }^{6}$ A. Bezryadin, C. N. Lau, and M. Tinkham, Nature (London) 404, 971 (2000).

${ }^{7}$ C. N. Lau, N. Markovic, M. Bockrath, A. Bezryadin, and M. Tinkham, Phys. Rev. Lett. 87, 217003 (2001).

${ }^{8}$ M. Tian, J. Wang, J. S. Kurtz, Y. Liu, M. H. W. Chan, T. S. Mayer, and T. E. Mallouk, Phys. Rev. B 71, 104521 (2005).

${ }^{9}$ M. Zgirski, K.-P. Riikonen, V. Touboltsev, and K. Arutyunov, Nano Lett. 5, 1029 (2005).

${ }^{10}$ A. Rogachev, A. T. Bollinger, and A. Bezryadin, Phys. Rev. Lett. 94, 017004 (2005). 
${ }^{11}$ F. Altomare, A. M. Chang, M. R. Melloch, Y. Hong, and C. W. Tu, Phys. Rev. Lett. 97, 017001 (2006); 98, 169901(E) (2007).

${ }^{12}$ A. T. Bollinger, A. Rogachev, and A. Bezryadin, Europhys. Lett. 76, 505 (2006).

${ }^{13}$ A. T. Bollinger, R. C. Dinsmore, A. Rogachev, and A. Bezryadin, arXiv:0707.4532 (unpublished).

${ }^{14}$ I. O. Kulik, Zh. Eksp. Teor. Fiz. 65, 2016 (1973) [Sov. Phys. JETP 38, 1008 (1974)].

${ }^{15}$ J. E. Mooij and G. Schön, Phys. Rev. Lett. 55, 114 (1985).

${ }^{16}$ A. D. Zaikin, D. S. Golubev, A. van Otterlo, and G. T. Zimányi, Phys. Rev. Lett. 78, 1552 (1997).

${ }^{17}$ S. Khlebnikov, Phys. Rev. Lett. 93, 090403 (2004); Phys. Rev. A 71, 013602 (2005).

${ }^{18}$ S. Khlebnikov and L. P. Pryadko, Phys. Rev. Lett. 95, 107007 (2005).

${ }^{19}$ X. G. Wen and A. Zee, Int. J. Mod. Phys. B 4, 437 (1990).
${ }^{20}$ D. Meidan, Y. Oreg, and G. Refael, Phys. Rev. Lett. 98, 187001 (2007).

${ }^{21}$ W. A. Little, Phys. Rev. 156, 396 (1967).

${ }^{22}$ J. S. Langer and V. Ambegaokar, Phys. Rev. 164, 498 (1967).

${ }^{23}$ D. E. McCumber and B. I. Halperin, Phys. Rev. B 1, 1054 (1970).

${ }^{24}$ J. Pearl, Appl. Phys. Lett. 5, 65 (1964).

${ }^{25}$ N. B. Kopnin and V. E. Kravtsov, Zh. Eksp. Teor. Fiz. 71, 1644 (1976) [Sov. Phys. JETP 44, 861 (1976)].

${ }^{26}$ H. P. Büchler, V. B. Geshkenbein, and G. Blatter, Phys. Rev. Lett. 92, 067007 (2004).

${ }^{27}$ S. Coleman, Phys. Rev. D 15, 2929 (1977); 16, 1248(E) (1977).

${ }^{28}$ D. S. Golubev and A. D. Zaikin, Phys. Rev. B 64, 014504 (2001).

${ }^{29}$ A. A. Abrikosov, L. P. Gorkov, and I. E. Dzyaloshinski, Methods of Quantum Field Theory in Statistical Physics (Dover, New York, 1975). 